\title{
Inflammation-Based Scores: A New Method for Patient-Targeted Strategies and Improved Perioperative Outcome in Cancer Patients
}

\author{
Dario Bugada, ${ }^{1,2}$ Massimo Allegri, ${ }^{2,3,4}$ Patricia Lavand'homme, ${ }^{5}$ \\ Marc De Kock, ${ }^{5}$ and Guido Fanelli ${ }^{2,6}$ \\ ${ }^{1}$ Department of Anaesthesia and Intensive Care, Foundation IRCCS Policlinico San Matteo, P.le Golgi 19, 27100 Pavia, Italy \\ ${ }^{2}$ SIMPAR (Study in Multidisciplinary Pain Research) Group, Italy \\ ${ }^{3}$ Department of Surgical, Medical, Diagnostic and Pediatric Science, University of Pavia, Via Aselli 45, 27100 Pavia, Italy \\ ${ }^{4}$ Pain Therapy Service, Foundation IRCCS Policlinico San Matteo, P.le Golgi 19, 27100 Pavia, Italy \\ ${ }^{5}$ Department of Anesthesia and Perioperative Medicine, Catholic University of Louvain, St. Luc Hospital, 10 Avenue Hippocrate, \\ 1200 Brussels, Belgium \\ ${ }^{6}$ Department of Anesthesia and Intensive Care, University of Parma, Via Gramsci 14, 43126 Parma, Italy
}

Correspondence should be addressed to Dario Bugada; dariobugada@gmail.com

Received 10 December 2013; Revised 10 March 2014; Accepted 19 March 2014; Published 27 April 2014

Academic Editor: Melchiorre Cervello

Copyright (c) 2014 Dario Bugada et al. This is an open access article distributed under the Creative Commons Attribution License, which permits unrestricted use, distribution, and reproduction in any medium, provided the original work is properly cited.

\begin{abstract}
Systemic inflammatory response (SIR) has actually been shown as an important prognostic factor associated with lower postoperative survival in several types of cancer. Thus, the challenge for physicians is to find specific, low-cost, and highlyreliable inflammatory markers, clearly correlated with prognosis and able to preoperatively stratify patient's risk. Inflammation is a promising target to improve perioperative outcome, and data show that anti-inflammation techniques have a great potential in the perioperative period of cancer surgery. Inflammation scores could be useful to stratify patients with a potential better response to anti-inflammation strategies. Furthermore, inflammation scores could prevent failure of clinical trials by a better definition of patients to be included in such trials; inflammation scoring could clarify the real role of different drugs and techniques on outcome after cancer surgery, defining if different therapies are required for different patients. The role of this review is to focus on the currently available scores, in order to clarify their rationale and to analyze the actual evidence and limits, providing physicians with an updated overview of the possible inflammation-based prognostic scores for cancer patients undergoing surgery.
\end{abstract}

\section{Introduction}

Inflammation plays a key role in cancer physiology, as it could promote carcinogenesis, dedifferentiation, and primary tumour growth [1]; furthermore, it promotes tumour cells proliferation by inhibiting apoptosis and increasing mitosis rate [1].

Inflammation has also some protective effects, participating in the initial anticancer response, mainly by cell-mediated immunity; immune cells can recognize factors produced by the inflammatory response in tumour to detect lymphocytes, macrophages, and dendritic cells (the so-called paradox of inflammation) [2]; inflammation has a causative role in many tumours and is a concomitant event in malignant recurrence [1].

As the role of inflammatory response is strictly connected with cancer physiology, many studies have investigated its role in cancer outcome. Regarding cancer surgery, systemic inflammatory response (SIR) has actually been shown as an important prognostic factor [3-57] associated with lower postoperative survival in several types of cancer.

Considering this prognostic value, the new challenges for physicians are represented by detecting degree of inflammation in each patient through specific inflammatory markers, clearly correlated with prognosis and able to preoperatively recognize immune changes for a better stratification of 
patient's risk. As inflammation is a complex phenomenon involving many cells and cytokines both systemically and locally (within the tumour), finding a low-cost, highly reliable, and easy to detect marker is often difficult. So far, a number of different prognostic scores based on inflammation have been proposed, with an increasing evidence of their value but with limits in their sensitivity and specificity. The role of this review is to focus on the currently studied and validated scores, in order to clarify their rationale and to analyze the actual evidence and limits, providing physicians with an updated overview of the possible inflammation-based prognostic scores for cancer patients undergoing surgery.

\section{Methods}

We systematically identified reports of studies assessing the use of inflammation-based scores. PubMed and MEDLINE databases were searched until November 2013 using the terms: "platelet to lymphocyte ratio" or "neutrophil to lymphocyte ratio" or "Glasgow prognostic score" and "cancer outcome." Only papers in English were considered. Additional reports were identified from reference lists of retrieved papers. Only full papers were considered.

Included studies were both prospective and retrospective; retrospective analyses of prospective data were included and listed as retrospective (Tables 2 and 3 ).

Principal outcome measures were overall survival, disease-free survival, time to recurrence, pathology-free survival, relapse-free survival, mortality, and tumor resectability.

We included studies in which a selected marker was demonstrated to have a prognostic value alone or in combination with other markers (not strictly connected with inflammation).

We included studies involving only human subjects, both surgical and nonsurgical patients.

\section{Results}

The initial electronic literature searches revealed 136 studies, and, after evaluation of the abstracts, 78 were identified as potentially meeting the inclusion criteria. Evaluated inflammatory markers were NLR (neutrophil to lymphocyte ratio), PLR (platelet to lymphocyte ratio), GPS (Glasgow prognostic scale), and mGPS (modified GPS). 58 studies were excluded because they were about (1) inflammation markers other than NLR, PLR, and (m) GPS, (2) cancer noninflammation markers, (3) prognostic but not inflammation-based scores specifically relating to a single type of tumor, (4) cancer survival associated with different chemotherapies, and (5) effect of "other" variables on patient's outcome (infections, BMI, cigarette smoke, nutritional status, depression, psychological interventions, different surgical approaches, hemodynamic instability, reoperations, and graft histologic patterns). One study was excluded because it was not in English, while one study was excluded because it was a poster abstract of a scientific meeting. 36 additional studies were identified from reference lists of retrieved papers; additional evaluated
TABLE 1: Glasgow Prognostic score and modified Glasgow Prognostic Score.

\begin{tabular}{lc}
\hline & Points \\
\hline GPS & \\
CRP $\leq 10 \mathrm{mg} / \mathrm{L}$ and albumin $\geq 35 \mathrm{~g} / \mathrm{L}$ & 1 \\
$\mathrm{CRP}>10 \mathrm{mg} / \mathrm{L}$ & 1 \\
Albumin $<35 \mathrm{~g} / \mathrm{L}$ & 2 \\
$\mathrm{CRP}>10 \mathrm{mg} / \mathrm{L}$ and albumin $<35 \mathrm{~g} / \mathrm{L}$ & \\
MODIFIED GPS & 0 \\
CRP $\leq 10 \mathrm{mg} / \mathrm{L}$ and albumin $\geq 35 \mathrm{~g} / \mathrm{L}$ & 1 \\
$\mathrm{CRP}>10 \mathrm{mg} / \mathrm{L}$ & 2 \\
$\mathrm{CRP}>10 \mathrm{mg} / \mathrm{L}$ and albumin $<35 \mathrm{~g} / \mathrm{L}$ &
\end{tabular}

CRP: C-Reactive Protein.

markers were COP-NLR and thrombocytosis. Finally, 114 studies were considered for this review. Regarding (m) GPS, we retrieved more studies than we list in the references part of this paper; we decided to cite only some of them because they are the most recent, and their results summarize what was demonstrated by previous published papers.

3.1. Glasgow Prognostic Score. Glasgow prognostic score (GPS) measures acute-phase protein markers of the SIR, namely, C-reactive protein and albumin using standard thresholds ( $>10 \mathrm{mg} / \mathrm{L}$ for C-reactive protein and $<35 \mathrm{~g} / \mathrm{L}$ for albumin), which were combined to form a cumulative inflammation-based prognostic score [58]. This was subsequently refined to form the modified Glasgow prognostic score (mGPS) [59] when, in patients with primary operable colorectal cancer, hypoalbuminemia alone was found to have the same prognostic value as a GPS of 0 (Table 1). The higher the score is, the higher the risk is; an increased mGPS was predictive of a reduced cancer-specific survival in all cancers [56].

GPS is considered as a measure for systemic inflammation and reflects some of the biological changes, in both immune response and nutritional status, associated with cancer patients. The connection between systemic inflammation and mGPS is mainly acted through interleukin 6 (IL-6) role, as it is a pleiotropic cytokine with many physiological actions. In fact, IL-6 promotes not only CRP upregulation, but also albumin downregulation in the liver, as well as protein synthesis [60] and thrombocytosis [61]; it is also important to underline that some of these characteristics are related to nutritional status, because an elevated CRP level, hypoalbuminemia, and low BMI reflect cachexia due to hypercytokinemia resulting from tumour progression.

The evidence that the inclusion of a leukocyte count may add prognostic value to the validated mGPS and the recent introduction of high-sensitivity $\mathrm{C}$-reactive protein measurements in routine clinical laboratory analysis (with threshold sensitivity lowered to $0.05 \mathrm{mg} / \mathrm{L}$ ) has further modified the C-reactive protein/albumin combination improving the prognostic value derived from the components of a differential leukocyte count (neutrophil, lymphocyte, and platelet counts), as demonstrated in a recent paper in which 


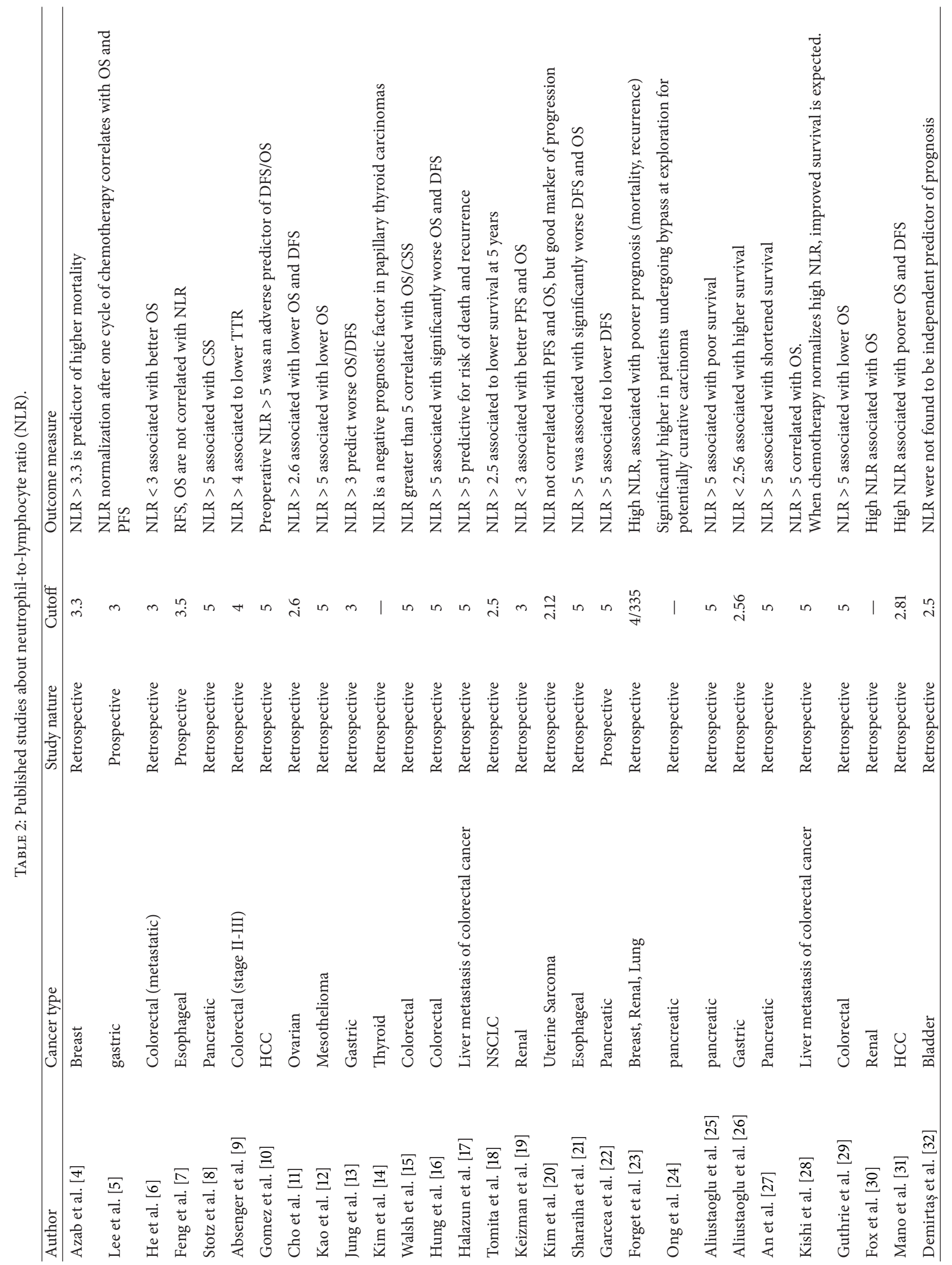




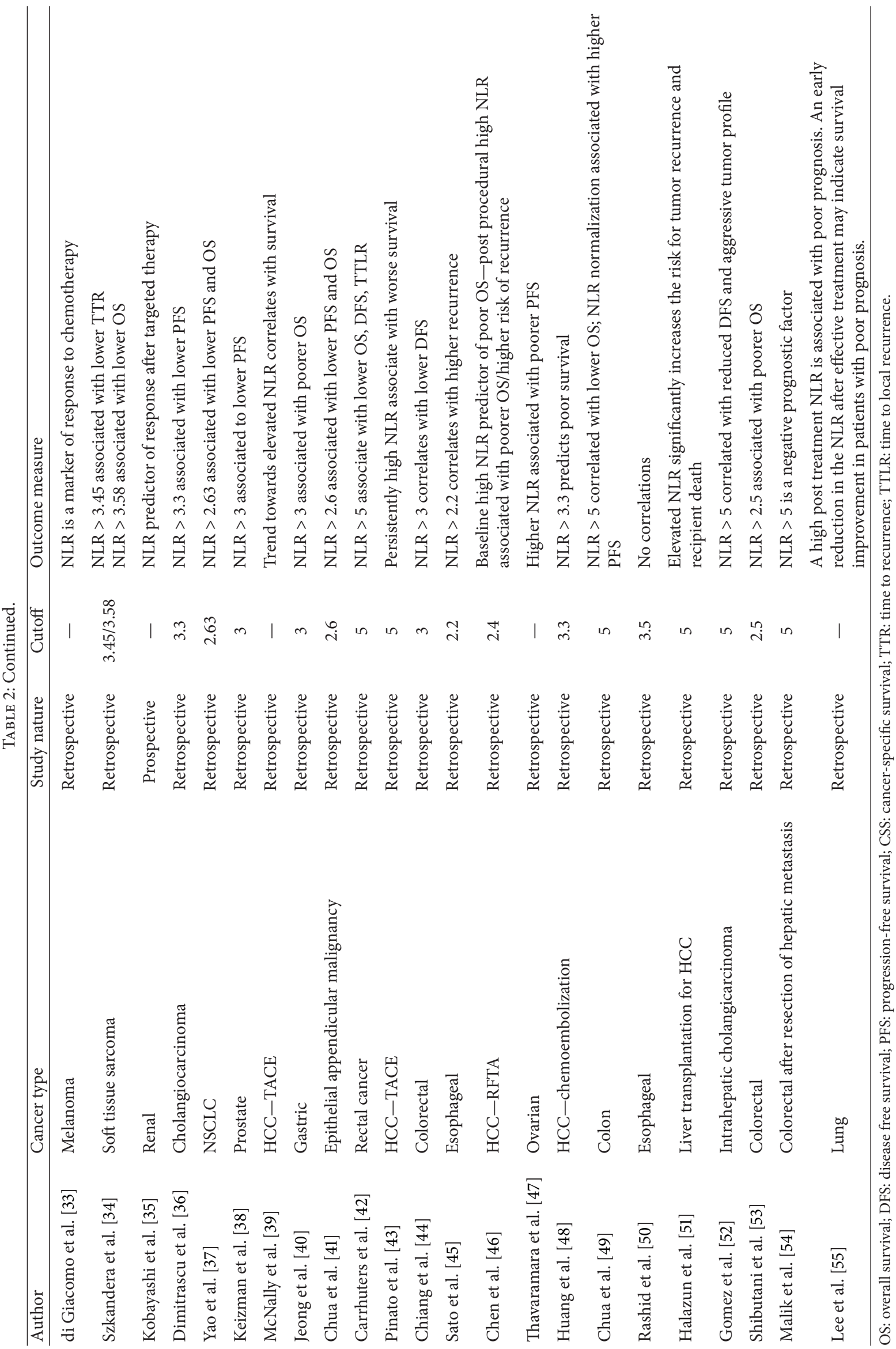




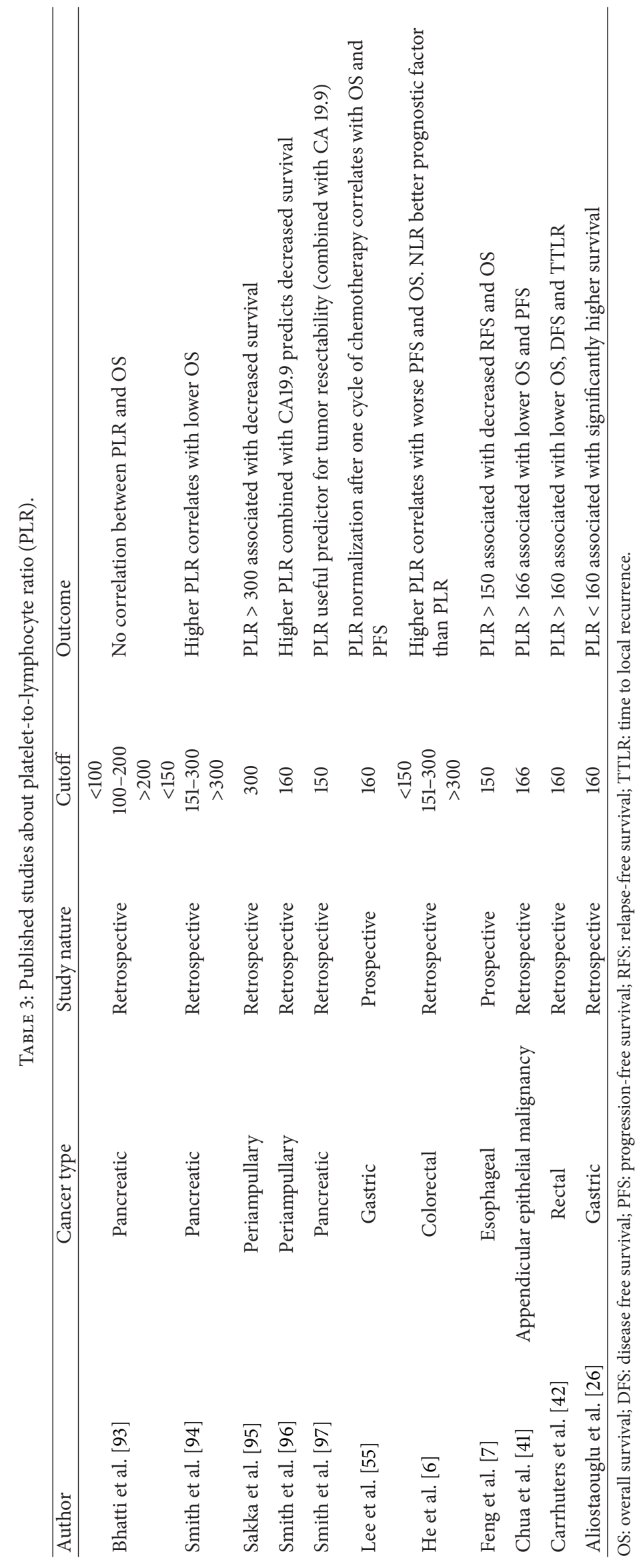


the addition of neutrophil and platelet counts, as well as a high-sensitivity C-reactive protein, enhanced the prognostic value of the mGPS [57].

GPS has been reported, in more than 60 studies $(>30,000$ patients), with independent prognostic value in patients with cancer in a heterogeneous variety of clinical scenarios and tumour types [62].

Currently, the GPS/mGPS is the most extensively validated one of the systemic inflammation-based prognostic scores and therefore may be used in the routine clinical assessment of patients with cancer, in addition or in preference to the current definitions of cachexia, together with tumour staging [62].

This biomarker not only identifies patients at risk for poorer prognosis but also provides a well-defined therapeutic target for treatment and future clinical trials. For example, patients with elevated mGPS scores should be considered in a precachexia status and offered with multimodal therapy (surgical tumour excision, anti-inflammation strategies, and metabolic and nutritional surveillance), which may delay the onset of cachexia and/or death [63]; anesthetic and surgical techniques able to reduce the enhanced inflammatory reaction after surgery could be planned in patients with higher basal inflammation. As a consequence, this will highlight the need to treat not only the tumour itself but also the SIR, a potentially more tractable target compared with well-established weight loss and/or poor performance status. Further studies are required to define the value of GPS/mGPS as a stratification factor, as selection criteria in randomized clinical trials, and as a therapeutic target in patients with cancer [62].

3.2. Neutrophil to Lymphocytes Ratio. Neutrophil to lymphocytes ratio (NLR) is one of the most studied inflammation prognostic markers for postoperative outcome. In fact, as it is well related to inflammation response, its role in identifying high-risk patients has been proposed in cancer and noncancer patients (see Table 2).

Different studies have highlighted the role of a high NLR as a preoperative tool to detect cancer patients with poorer prognosis, in terms of both general comorbidities and cancer disease-free and overall survival [64]. Furthermore, some studies have identified NLR as a valid tool to identify patients who are more sensitive to specific chemotherapy regimen both in the surgical and nonsurgical setting $[5,16,28,33,35$, 45, 55].

NLR is simple and reliable, being part of the standard exams for clinical evaluation, and can be assessed both preoperatively and postoperatively. Even if the majority of studies evaluate this marker in the preoperative setting, its prognostic value has been associated also with postoperative value, in terms of short-term morbidity and long-term mortality [20, $23,28,39,43,46,49]$.

The relation between NLR and cancer outcome is probably to be found in tumour-associated immune changes; an elevated NLR reflects a decreased lymphocyte-mediated immunity (with an alteration in CD4+ helper/CD8+ suppressors ratio) and an increased production of inflammatory agents such as vascular endothelial growth factor (VEGF) that promotes tumour growth [65]; some studies evidence that the highest values of NLR are associated with aggressive tumour profiles [52]. Other studies focus on the NLR trend during tumour history, showing that a persistently high value is associated with poorer outcome (Table 2); all these findings suggest a probable direct connection between cancer biology and systemic inflammation expressed by NLR.

Even though most of the studies are retrospective, they reach the same conclusions. There are currently few prospective studies assessing the prognostic value of NLR, and a lack of homogeneity still exists in the definition of a standard NLR cutoff associated with different prognoses (Table 2); maybe different cutoffs have to be established for different types of cancer, as each type of cancer is associated with different changes in immune response.

3.3. The Role of Reactive Thrombocytosis: Platelet Count, COPNLR, and Platelet to Lymphocyte Ratio. Recent studies have demonstrated that reactive thrombocytosis is associated with lower survival after surgery for several types of cancer [6669], and platelet count could be related to the SIR, even if it is not still known which is the exact link between platelet, inflammation, and cancer outcomes. Reactive thrombocytosis is induced in a background of hypercytokinemia related to tumour progression. Among several inflammatory cytokines, IL-6 plays an important role in reactive thrombocytosis [61]. IL-6 has a cell-proliferative effect, triggering the differentiation of megakaryocytes to platelets in the bone marrow $[70,71]$.

Although the normal platelet count is $15-30 \times 10^{4} \mathrm{~mm}^{3}$, the cutoff value for reactive thrombocytosis is not clearly defined. However, most previous studies have used a cutoff value of $30-40 \times 10^{4} \mathrm{~mm}^{3}[72,73]$. More studies are needed also to confirm the validity of this cutoff value.

Thrombocytosis is also induced from the tumour itself [74]. Generally, thrombocytosis is a laboratory finding in 10$57 \%$ of patients with malignancy, as a variety of neoplastic cells can stimulate platelet activation [75, 76]; some studies have revealed that cancer cells secrete vascular endothelial growth factor (VEGF), which also stimulates megakaryocyte differentiation [77]. Because VEGF induction promotes tumour growth $[74,78]$, thrombocytosis indirectly reflects tumour progression; a high level of VEGF is found in serum, platelets, and leukocytes of patients with malignant disease [79], and platelet interactions with malignant cells promote metastasis [80].

Shimada et al. [69] have reported an association between high NLR and high platelet count in gastric cancer prognosis and also found that reactive thrombocytosis [81] was associated with lower postoperative survival in patients with esophageal cancer. Other studies confirmed a high platelet count as a negative prognostic factor for renal cell, pancreatic, and colorectal cancers [72, 73, 82-84].

These results gave strong support to the use of a combination of reactive thrombocytosis and the NLR for prediction of postoperative survival; in a recent work by Ishizuka et al. [85] presented the COP-NLR (combination of olatelet count 
and neutrophil to lymphocyte ratio), for predicting the postoperative survival of patients with colorectal cancer. In their study patients were divided in 3 groups based on presence or absence of platelet count $>300.000 / \mathrm{mm}^{3}$ and NLR $>3$ and a statistically significant difference in cancer specific survival were retrieved, with COP-NLR being able to classify patient's outcome into three independent groups. Moreover, COPNLR resulted associated with tumour-related characteristics (type and dimension, invasivity and metastasis, operative curability, CEA) and SIR-related characteristics (high CRP, hypoalbuminemia, low BMI, and high NLR). These findings suggest the new COP-NLR as a new inflammation-based prognostic marker to complement classical tumour staging, reflecting biological changes related to high levels of IL-6 (the theoretically ideal marker of tumour-related SIR, whose serum levels are often difficult to be measured in the clinical practice). As there is only this retrospective study, more data are needed to confirm its predictive value demonstrating its specificity in other types of cancer. Nevertheless COP-NLR seems a valuable and promising tool for patient stratification in colorectal cancer.

Another prognostic marker related to platelet proliferation is the platelet to lymphocyte ratio (PLR).

PLR has been identified as a prognostic marker in patients with advanced gastric cancer [55] treated with chemotherapy (identifying more sensitive patients to specific type of chemotherapy), and its preoperative value correlated with prognosis (Table 3). Like NLR, studies are mainly retrospective and about only a few types of cancer; moreover, a lack of homogeneity still exists in the definition of a standard PLR cutoff associated with different prognoses. Further studies are needed to understand the real role of this marker and if it has a higher or lower prognostic value relating to NLR $[6,7]$.

\section{Discussion}

As inflammation is strictly related to cancer, SIR is demonstrated to influence cancer patients' outcome.

In the last decade research focused its attention on finding specific biomarkers able to quantify systemic inflammation, in order to stratify patients' risk and detect patients more prone to be treated with different therapies, especially in cancer patients undergoing surgery.

These markers are not always simple or cheap to measure, so efforts have been done to find a low-cost, highly reliable, and easy to detect biomarker, clearly associated with prognosis and easily evaluated with routine laboratory analysis. Existing evidence suggests that a higher prognostic value is associated not with a single marker but with a combination of them; inflammation-based scores reflect many biological changes connected with cancer, and the impact of systemic inflammation on various aspects of patient's physiology [86, 87].

Among these inflammation-based scores, GPS is the most extensively validated one. It may be used in the routine clinical assessment of patients with cancer (particularly due to its strict connection with cachexia and poor performance status), suggesting that inflammation is a further therapeutic target, potentially able to delay the onset of cachexia and/or death in cancer patients.

Other scores reflect cancer-associated immune changes focusing on specific mechanisms connected with hostdefense and tumour progression (NLR, thrombocytosis); regarding NLR, initial findings about a correlation with tumour aggressiveness were retrieved [52], and a possible role not only of the preoperative value itself, but also of the whole NLR trend during patient history was observed $[28,39,43,46,49]$, suggesting a possible role as a follow-up marker (to be demonstrated in proper designed trials).

Different evidence is available for each of them as prognostic scores, but limits still exist.

Firstly, most of the studies are retrospective, and prospective evaluations are needed to confirm the literature data. Moreover, a better definition of cutoff values has to be pursued; actual values are very heterogeneous and sometimes differ in the same study between different centers [23]; finally, it is still to be defined if different cancers are associated with different cutoffs or there is a common marker for prognosis to be used for all types of tumour. Investigators have just exceptionally compared effectiveness of different scores on predicting prognosis [6,7]; however, further and more homogeneous studies are warranted to understand which score is more efficient in predicting the level of systemic inflammation and prognosis in each specific type of cancer, also according to tumour stage.

However, despite limits, there is a rising evidence of the validity of prognostic inflammation-based scores and biomarkers. Their analysis allowed stratifying the subgroup of patients and to understand cancer-related physiological mechanisms [87], configuring the first step towards a better multimodal therapy that, apart from cancer eradication, takes into account other targets to prolong patients' survival.

Inflammation is a promising target to improve perioperative outcome, and data show that anti-inflammatory techniques have a great potential in the perioperative period of cancer surgery [88]; evidence exists for a higher cytokine activation in vitro in patients with higher BMI (proinflammatory condition) [89]. The ability to quantify inflammation could allow identifying patients more at risk of enhanced response and hypercytokinemia in the postoperative period; inflammation scores could be useful to stratify patients more at risk or with a potential better response to antiinflammatory strategies (and more worth to be treated with them), considering that the effect of NSAIDs and potentially all other anti-inflammatory techniques may depend on systemic inflammation level.

Regarding research, inflammatory scores could prevent failure of clinical trials testing drug candidate [90] and minimize unuseful exposure to ineffective therapies [90]. Inflammation scoring could clarify the real role of different drugs and techniques on outcome after cancer surgery, defining if different therapies are required for different patients. Forget et al. [23] showed that a single intraoperative administration of NSAIDs (ketorolac and diclofenac) could counteract effects of inflammation on cancer outcome and that this effect is twice greater in patients with NLR $>4$ than in the whole series. Conversely, tumours with a slow growth, typically with 
a low level of inflammation and a small risk of early relapse, could have a smaller benefit of NSAIDs. This observation was done in a large retrospective series of 1,111 prostate cancer patients [91] and highlights the possible ability for NLR to guide drug choice on a specific target of patients. If data will be confirmed (NCT01806259) we could have indication to use NSAIDs on subtypes of patients in order to improve oncological outcome.

Moreover, discrepancies between studies focusing on the effects of regional analgesia or morphine in the perioperative period exist [92]; this could be explained by their different efficacy in patients with different systemic inflammation. Inclusion of inflammation analysis in future clinical trials could clarify which patients are more indicated to be treated with regional techniques.

Finally, as opioids were advocated to have immunodeppressive action, able to facilitate tumour dissemination [92], it would be useful to identify patients with enhanced inflammatory response, in order to understand if opioid therapy has beneficial effects on outcome in this specific subgroup of patients.

\section{Conflict of Interests}

The authors declare that there is no conflict of interests regarding the publication of this paper.

\section{Acknowledgment}

This paper was funded by a grant from the Italian Health Ministry, Project of "New nanotechnology and biomedical approaches to improve postoperative pain treatment reducing risks related to opioids", Project code: GR-2010-2318370.

\section{References}

[1] S. Demaria, E. Pikarsky, M. Karin et al., "Cancer and inflammation: promise for biologic therapy," Journal of Immunotherapy, vol. 33, no. 4, pp. 335-351, 2010.

[2] C. A. Dinarello, "The paradox of pro-inflammatory cytokines in cancer," Cancer and Metastasis Reviews, vol. 25, no. 3, pp. 307313, 2006.

[3] P. Forget, P. Coulie, M. Retsky, R. Demicheli, J.-P. Machiels, and M. de Kock, "Is there a rationale for an anaesthesiologist's role against cancer recurrence?" Acta Anaesthesiologica Belgica, vol. 64, no. 1, pp. 15-24, 2013.

[4] B. Azab, V. R. Bhatt, J. Phookan et al., "Usefulness of the neutrophil-to-lymphocyte ratio in predicting short- and longterm mortality in breast cancer patients," Annals of Surgical Oncology, vol. 19, no. 1, pp. 217-224, 2012.

[5] S. Lee, S. Y. Oh, S. H. Kim et al., "Prognostic significance of neutrophil lymphocyte ratio and platelet lymphocyte ratio in advanced gastric cancer patients treated with FOLFOX chemotherapy," BMC Cancer, vol. 13, article 350, 2013.

[6] W. He, C. Yin, G. Guo et al., "Initial neutrophil lymphocyte ratio is superior to platelet lymphocyte ratio as an adverse prognostic and predictive factor in metastatic colorectal cancer," Medical Oncology, vol. 30, no. 1, article 439, 2013.

[7] J. F. Feng, Y. Huang, Q. Zhao, and Q. X. Chen, "Clinical significance of preoperative neutrophil lymphocyte ratio versus platelet lymphocyte ratio in patients with small cell carcinoma of the esophagus," The Scientific World Journal, vol. 2013, Article ID 504365, 7 pages, 2013.

[8] M. Stotz, A. Gerger, F. Eisner et al., "Increased neutrophillymphocyte ratio is a poor prognostic factor in patients with primary operable and inoperable pancreatic cancer," British Journal of Cancer, vol. 109, no. 2, pp. 416-421, 2013.

[9] G. Absenger, J. Szkandera, M. Stotz et al., "Preoperative neutrophil-to-lymphocyte ratio predicts clinical outcome in patients with stage II and III colon cancer," Anticancer Research, vol. 33, no. 10, pp. 4591-4594, 2013.

[10] D. Gomez, S. Farid, H. Z. Malik et al., "Preoperative neutrophilto-lymphocyte ratio as a prognostic predictor after curative resection for hepatocellular carcinoma," World Journal of Surgery, vol. 32, no. 8, pp. 1757-1762, 2008.

[11] H. Cho, H. W. Hur, S. W. Kim et al., "Pre-treatment neutrophil to lymphocyte ratio is elevated in epithelial ovarian cancer and predicts survival after treatment," Cancer Immunology, Immunotherapy, vol. 58, no. 1, pp. 15-23, 2009.

[12] S. C. H. Kao, N. Pavlakis, R. Harvie et al., "High blood neutrophil-to-lymphocyte ratio is an indicator of poor prognosis in malignant mesothelioma patients undergoing systemic therapy," Clinical Cancer Research, vol. 16, no. 23, pp. 5805-5813, 2010.

[13] M. R. Jung, Y. K. Park, O. Jeong et al., "Elevated preoperative neutrophil to lymphocyte ratio predicts poor survival following resection in late stage gastric cancer," Journal of Surgical Oncology, vol. 104, no. 5, pp. 504-510, 2011.

[14] J. Y. Kim, T. Park, S. H. Jeong et al., "Prognostic importance of baseline neutrophil to lymphocyte ratio in patients with advanced papillary thyroid carcinomas," Endocrine, 2013.

[15] S. R. Walsh, E. J. Cook, F. Goulder, T. A. Justin, and N. J. Keeling, "Neutrophil-lymphocyte ratio as a prognostic factor in colorectal cancer," Journal of Surgical Oncology, vol. 91, no. 3, pp. 181-184, 2005.

[16] H.-Y. Hung, J.-S. Chen, C. Y. Yeh et al., "Effect of preoperative neutrophil-lymphocyte ratio on the surgical outcomes of stage II colon cancer patients who do not receive adjuvant chemotherapy," International Journal of Colorectal Disease, vol. 26, no. 8, pp. 1059-1065, 2011.

[17] K. J. Halazun, A. Aldoori, H. Z. Malik et al., "Elevated preoperative neutrophil to lymphocyte ratio predicts survival following hepatic resection for colorectal liver metastases," European Journal of Surgical Oncology, vol. 34, no. 1, pp. 55-60, 2008.

[18] M. Tomita, T. Shimizu, T. Ayabe, A. Yonei, and T. Onitsuka, "Preoperative neutrophil to lymphocyte ratio as a prognostic predictor after curative resection for non-small cell lung cancer," Anticancer Research, vol. 31, no. 9, pp. 2995-2998, 2011.

[19] D. Keizman, M. Ish-Shalom, P. Huang et al., "The association of pre-treatment neutrophil to lymphocyte ratio with response rate, progression free survival and overall survival of patients treated with sunitinib for metastatic renal cell carcinoma," European Journal of Cancer, vol. 48, no. 2, pp. 202-208, 2012.

[20] H. S. Kim, K. H. Han, H. H. Chung et al., "Neutrophil to lymphocyte ratio for preoperative diagnosis of uterine sarcomas: a case-matched comparison," European Journal of Surgical Oncology, vol. 36, no. 7, pp. 691-698, 2010.

[21] R. Z. Sharaiha, K. J. Halazun, F. Mirza et al., "Elevated preoperative neutrophil: lymphocyte ratio as a predictor of postoperative disease recurrence in esophageal cancer," Annals of Surgical Oncology, vol. 18, no. 12, pp. 3362-3369, 2011. 
[22] G. Garcea, N. Ladwa, C. P. Neal, M. S. Metcalfe, A. R. Dennison, and D. P. Berry, "Preoperative neutrophil-to-lymphocyte ratio (NLR) is associated with reduced disease-free survival following curative resection of pancreatic adenocarcinoma," World Journal of Surgery, vol. 35, no. 4, pp. 868-872, 2011.

[23] P. Forget, J. P. Machiels, P. G. Coulie et al., "Neutrophil: lymphocyte ratio and intraoperative use of ketorolac or diclofenac are prognostic factors in different cohorts of patients undergoing breast, lung, and kidney cancer surgery," Annals of Surgical Oncology, vol. 20, supplement 3, pp. S650-S660, 2013.

[24] S. L. Ong, G. Garcea, S. C. Thomasset et al., "Surrogate markers of resectability in patients undergoing exploration of potentially resectable pancreatic adenocarcinoma," Journal of Gastrointestinal Surgery, vol. 12, no. 6, pp. 1068-1073, 2008.

[25] M. Aliustaoglu, A. Bilici, M. Seker et al., "The association of pretreatment peripheral blood markers with survival in patients with pancreatic cancer," Hepato-Gastroenterology, vol. 57, no. 99-100, pp. 640-645, 2010.

[26] M. Aliustaoglu, A. Bilici, B. B. O. Ustaalioglu et al., "The effect of peripheral blood values on prognosis of patients with locally advanced gastric cancer before treatment," Medical Oncology, vol. 27, no. 4, pp. 1060-1065, 2010.

[27] X. An, P.-R. Ding, Y.-H. Li et al., "Elevated neutrophil to lymphocyte ratio predicts survival in advanced pancreatic cancer," Biomarkers, vol. 15, no. 6, pp. 516-522, 2010.

[28] Y. Kishi, S. Kopetz, Y. S. Chun, M. Palavecino, E. K. Abdalla, and J.-N. Vauthey, "Blood neutrophil-to-lymphocyte ratio predicts survival in patients with colorectal liver metastases treated with systemic chemotherapy," Annals of Surgical Oncology, vol. 16, no. 3, pp. 614-622, 2009.

[29] G. J. Guthrie, C. S. Roxburgh, O. M. Farhan-Alanie, P. G. Horgan, and D. C. McMillan, "Comparison of the prognostic value of longitudinal measurements of systemic inflammation in patients undergoing curative resection of colorectal cancer," British Journal of Cancer, vol. 109, no. 1, pp. 24-28, 2013.

[30] P. Fox, M. Hudson, C. Brown et al., "Markers of systemic inflammation predict survival in patients with advanced renal cell cancer," British Journal of Cancer, vol. 109, no. 1, pp. 147-153, 2013.

[31] Y. Mano, K. Shirabe, Y. Yamashita et al., "Preoperative neutrophil-to-lymphocyte ratio is a predictor of survival after hepatectomy for hepatocellular carcinoma: a retrospective analysis," Annals of Surgery, vol. 258, no. 2, pp. 301-305, 2013.

[32] A. Demirtaş, V. Sabur, E. C. Akınsal et al., "Can neutrophillymphocyte ratio and lymph node density be used as prognostic factors in patients undergoing radical cystectomy?" The Scientific World Journal, vol. 2013, Article ID 703579, 5 pages, 2013.

[33] A. M. di Giacomo, L. Calabrò, R. Danielli et al., "Longterm survival and immunological parameters in metastatic melanoma patients who responded to ipilimumab $10 \mathrm{mg} / \mathrm{kg}$ within an expanded access programme," Cancer Immunology, Immunotherapy, vol. 62, no. 6, pp. 1021-1028, 2013.

[34] J. Szkandera, G. Absenger, B. Liegl-Atzwanger et al., "Elevated preoperative neutrophil/lymphocyte ratio is associated with poor prognosis in soft-tissue sarcoma patients," British Journal of Cancer, vol. 108, no. 8, pp. 1677-1683, 2013.

[35] M. Kobayashi, T. Kubo, K. Komatsu et al., "Changes in peripheral blood immune cells: their prognostic significance in metastatic renal cell carcinoma patients treated with molecular targeted therapy," Medical Oncology, vol. 30, no. 2, article 556, 2013.
[36] T. Dumitrascu, D. Chirita, M. Ionescu, and I. Popescu, "Resection for hilar cholangiocarcinoma: analysis of prognostic factors and the impact of systemic inflammation on long-term outcome," Journal of Gastrointestinal Surgery, vol. 17, no. 5, pp. 913924, 2013.

[37] Y. Yao, D. Yuan, H. Liu, X. Gu, and Y. Song, "Pretreatment neutrophil to lymphocyte ratio is associated with response to therapy and prognosis of advanced non-small cell lung cancer patients treated with first-line platinum-based chemotherapy," Cancer Immunology, Immunotherapy, vol. 62, no. 3, pp. 471-479, 2013.

[38] D. Keizman, M. Gottfried, M. Ish-Shalom et al., "Pretreatment neutrophil-to-lymphocyte ratio in metastatic castrationresistant prostate cancer patients treated with ketoconazole: association with outcome and predictive nomogram," The Oncologist, vol. 17, no. 12, pp. 1508-1514, 2012.

[39] M. E. McNally, A. Martinez, H. Khabiri et al., "Inflammatory markers are associated with outcome in patients with unresectable hepatocellular carcinoma undergoing transarterial chemoembolization," Annals of Surgical Oncology, vol. 20, no. 3, pp. 923-928, 2013.

[40] J. H. Jeong, S. M. Lim, J. Y. Yun et al., "Comparison of two inflammation-based prognostic scores in patients with unresectable advanced gastric cancer," Oncology, vol. 83, no. 5, pp. 292-299, 2012.

[41] T. C. Chua, C. H. Chong, W. Liauw, J. Zhao, and D. L. Morris, "Inflammatory markers in blood and serum tumor markers predict survival in patients with epithelial appendiceal neoplasms undergoing surgical cytoreduction and intraperitoneal chemotherapy," Annals of Surgery, vol. 256, no. 2, pp. 342-349, 2012.

[42] R. Carruthers, L. M. Tho, J. Brown, S. Kakumanu, E. McCartney, and A. C. McDonald, "Systemic inflammatory response is a predictor of outcome in patients undergoing preoperative chemoradiation for locally advanced rectal cancer," Colorectal Disease, vol. 14, no. 10, pp. e701-e707, 2012.

[43] D. J. Pinato and R. Sharma, "An inflammation-based prognostic index predicts survival advantage after transarterial chemoembolization in hepatocellular carcinoma," Translational Research, vol. 160, no. 2, pp. 146-152, 2012.

[44] S.-F. Chiang, H.-Y. Hung, R. Tang et al., "Can neutrophilto-lymphocyte ratio predict the survival of colorectal cancer patients who have received curative surgery electively?" International Journal of Colorectal Disease, vol. 27, no. 10, pp. 13471357, 2012.

[45] H. Sato, Y. Tsubosa, and T. Kawano, "Correlation between the pretherapeutic neutrophil to lymphocyte ratio and the pathologic response to neoadjuvant chemotherapy in patients with advanced esophageal cancer," World Journal of Surgery, vol. 36, no. 3, pp. 617-622, 2012.

[46] T.-M. Chen, C.-C. Lin, P.-T. Huang, and C.-F. Wen, "Neutrophilto-lymphocyte ratio associated with mortality in early hepatocellular carcinoma patients after radiofrequency ablation," Journal of Gastroenterology and Hepatology, vol. 27, no. 3, pp. 553-561, 2012.

[47] T. Thavaramara, C. Phaloprakarn, S. Tangjitgamol, and S. Manusirivithaya, "Role of neutrophil to lymphocyte ratio as a prognostic indicator for epithelial ovarian cancer," The Journal of the Medical Association of Thailand, vol. 94, no. 7, pp. 871-877, 2011.

[48] Z.-L. Huang, J. Luo, M.-S. Chen, J.-Q. Li, and M. Shi, "Blood neutrophil-to-lymphocyte ratio predicts survival in 
patients with unresectable hepatocellular carcinoma undergoing transarterial chemoembolization," Journal of Vascular and Interventional Radiology, vol. 22, no. 5, pp. 702-709, 2011.

[49] W. Chua, K. A. Charles, V. E. Baracos, and S. J. Clarke, "Neutrophil/lymphocyte ratio predicts chemotherapy outcomes in patients with advanced colorectal cancer," British Journal of Cancer, vol. 104, no. 8, pp. 1288-1295, 2011.

[50] F. Rashid, N. Waraich, I. Bhatti et al., "A pre-operative elevated neutrophil: lymphocyte ratio does not predict survival from oesophageal cancer resection," World Journal of Surgical Oncology, vol. 8, article 1, 2010.

[51] K. J. Halazun, M. A. Hardy, A. A. Rana et al., "Negative impact of neutrophil-lymphocyte ratio on outcome after liver transplantation for hepatocellular carcinoma," Annals of Surgery, vol. 250, no. 1, pp. 141-151, 2009.

[52] D. Gomez, G. Morris-Stiff, G. J. Toogood, J. P. A. Lodge, and K. R. Prasad, "Impact of systemic inflammation on outcome following resection for intrahepatic cholangiocarcinoma," Journal of Surgical Oncology, vol. 97, no. 6, pp. 513-518, 2008.

[53] M. Shibutani, K. Maeda, H. Nagahara et al., "A high preoperative neutrophil-to-lymphocyte ratio is associated with poor survival in patients with colorectal cancer," Anticancer Research, vol. 33, no. 8, pp. 3291-3294, 2013.

[54] H. Z. Malik, K. R. Prasad, K. J. Halazun et al., "Preoperative prognostic score for predicting survival after hepatic resection for colorectal liver metastases," Annals of Surgery, vol. 246, no. 5, pp. 806-814, 2007.

[55] Y. Lee, S. H. Kim, J. Y. Han, H. T. Kim, T. Yun, and J. S. Lee, "Early neutrophil-to-lymphocyte ratio reduction as a surrogate marker of prognosis in never smokers with advanced lung adenocarcinoma receiving gefitinib or standard chemotherapy as first-line therapy," Journal of Cancer Research and Clinical Oncology, vol. 138, no. 12, pp. 2009-2016, 2012.

[56] M. J. Proctor, D. S. Morrison, D. Talwar et al., "An inflammationbased prognostic score (mGPS) predicts cancer survival independent of tumour site: a Glasgow Inflammation Outcome Study," British Journal of Cancer, vol. 104, no. 4, pp. 726-734, 2011.

[57] M. J. Proctor, P. G. Horgan, D. Talwar, C. D. Fletcher, D. S. Morrison, and D. C. McMillan, "Optimization of the systemic inflammation-based Glasgow prognostic score: a Glasgow Inflammation Outcome Study," Cancer, vol.119, no. 12, pp. 23252332, 2013.

[58] L. M. Forrest, D. C. McMillan, C. S. McArdle, W. J. Angerson, and D. J. Dunlop, "Evaluation of cumulative prognostic scores based on the systemic inflammatory response in patients with inoperable non-small-cell lung cancer," British Journal of Cancer, vol. 89, no. 6, pp. 1028-1030, 2003.

[59] D. C. McMillan, J. E. M. Crozier, K. Canna, W. J. Angerson, and C. S. McArdle, "Evaluation of an inflammation-based prognostic score (GPS) in patients undergoing resection for colon and rectal cancer," International Journal of Colorectal Disease, vol. 22, no. 8, pp. 881-886, 2007.

[60] G. Ramadori, J. van Damme, H. Rieder, and K.-H. M. zum Buschenfelde, "Interleukin 6, the third mediator of acute-phase reaction, modulates hepatic protein synthesis in human and mouse. Comparison with interleukin $1 \beta$ and tumor necrosis factor- $\alpha$," European Journal of Immunology, vol. 18, no. 8, pp. 1259-1264, 1988.

[61] E. Sierko and M. Z. Wojtukiewicz, "Platelets and angiogenesis in malignancy," Seminars in Thrombosis \& Hemostasis, vol. 30, no. 1, pp. 95-108, 2004.
[62] D. C. McMillan, "The systemic inflammation-based Glasgow prognostic score: a decade of experience in patients with cancer," Cancer Treatment Reviews, vol. 39, no. 5, pp. 534-540, 2013.

[63] N. MacDonald, "Terminology in cancer cachexia: importance and status," Current Opinion in Clinical Nutrition and Metabolic Care, vol. 15, no. 3, pp. 220-225, 2012.

[64] G. J. Guthrie, K. A. Charles, C. S. Roxburgh, P. G. Horgan, D. C. McMillan, and S. J. Clarke, "The systemic inflammationbased neutrophil-lymphocyte ratio: experience in patients with cancer," Critical Reviews in Oncology/Hematology, vol. 88, no. 1, pp. 218-230, 2013.

[65] C. Fondevila, J. P. Metges, J. Fuster et al., "p53 and VEGF expression are independent predictors of tumour recurrence and survival following curative resection of gastric cancer," British Journal of Cancer, vol. 90, no. 1, pp. 206-215, 2004.

[66] M. Monreal, J. Fernandez-Llamazares, M. Piñol et al., "Platelet count and survival in patients with colorectal cancer-a preliminary study," Thrombosis and Haemostasis, vol. 79, no. 5, pp. $916-$ 918, 1998.

[67] Y. Takahashi, C. D. Bucana, Y. Akagi et al., "Significance of platelet-derived endothelial cell growth factor in the angiogenesis of human gastric cancer," Clinical Cancer Research, vol. 4, no. 2, pp. 429-434, 1998.

[68] T. Browder, J. Folkman, and S. Pirie-Shepherd, "The hemostatic system as a regulator of angiogenesis," The Journal of Biological Chemistry, vol. 275, no. 3, pp. 1521-1524, 2000.

[69] H. Shimada, N. Takiguchi, O. Kainuma et al., "High preoperative neutrophil-lymphocyte ratio predicts poor survival in patients with gastric cancer," Gastric Cancer, vol. 13, no. 3, pp. 170-176, 2010.

[70] T. Imai, K. Koike, T. Kubo et al., "Interleukin-6 supports human megakaryocytic proliferation and differentiation in vitro," Blood, vol. 78, no. 8, pp. 1969-1974, 1991.

[71] F. W. Ruscetti, "Hematologic effects of interleukin-1 and interleukin-6," Current Opinion in Hematology, vol. 1, no. 3, pp. 210-215, 1994.

[72] M. Ishizuka, H. Nagata, K. Takagi, Y. Iwasaki, and K. Kubota, "Preoperative thrombocytosis is associated with survival after surgery for colorectal cancer," Journal of Surgical Oncology, vol. 106, no. 7, pp. 887-891, 2012.

[73] N. P. Symbas, M. F. Townsend, R. El-Galley, T. E. Keane, S. D. Graham, and J. A. Petros, "Poor prognosis associated with thrombocytosis in patients with renal cell carcinoma," British Journal of Urology International, vol. 86, no. 3, pp. 203-207, 2000.

[74] A. A. Khorana and R. L. Fine, "Pancreatic cancer and thromboembolic disease," The Lancet Oncology, vol. 5, no. 11, pp. 655663, 2004.

[75] M. L. George, S. A. Eccles, M. G. Tutton, A. M. Abulafi, and R. I. Swift, "Correlation of plasma and serum vascular endothelial growth factor levels with platelet count in colorectal cancer: clinical evidence of platelet scavenging?" Clinical Cancer Research, vol. 6, no. 8, pp. 3147-3152, 2000.

[76] E. Gunsilius, A. Petzer, G. Stockhammer et al., “Thrombocytes ave the major source for soluble vascular endothelial growth factor in peripheral blood," Oncology, vol. 58, no. 2, pp. 169-174, 2000.

[77] M. Troxler, K. Dickinson, and S. Homer-Vanniasinkam, "Platelet function and antiplatelet therapy," British Journal of Surgery, vol. 94, no. 6, pp. 674-682, 2007. 
[78] C. Gorelick, V. Andikyan, M. MacK, Y.-C. Lee, and O. Abulafia, "Prognostic significance of preoperative thrombocytosis in patients with endometrial carcinoma in an inner-city population," International Journal of Gynecological Cancer, vol. 19, no. 8, pp. 1384-1389, 2009.

[79] O. Lavie, G. Comerci, V. Daras, B. S. Bolger, A. Lopes, and J. M. Monaghan, "Thrombocytosis in women with vulvar carcinoma," Gynecologic Oncology, vol. 72, no. 1, pp. 82-86, 1999.

[80] T. Gislason and E. Nou, "Sedimentation rate, leucocytes, platelet count and haemoglobin in bronchial carcinoma: an epidemiological study," European Journal of Respiratory Diseases, vol. 66, no. 2, pp. 141-146, 1985.

[81] H. Shimada, G. Oohira, S.-I. Okazumi et al., "Thrombocytosis associated with poor prognosis in patients with esophageal carcinoma," Journal of the American College of Surgeons, vol. 198, no. 5, pp. 737-741, 2004.

[82] R. E. Schwarz and H. Keny, "Preoperative platelet count predicts survival after resection of periampullary adenocarcinoma," Hepato-Gastroenterology, vol. 48, no. 41, pp. 1493-1498, 2001.

[83] K. Suzuki, K. Aiura, M. Kitagou et al., "Platelets counts closely correlate with the disease-free survival interval of pancreatic cancer patients," Hepato-Gastroenterology, vol. 51, no. 57, pp. 847-853, 2004.

[84] K. M. Brown, C. Domin, G. V. Aranha, S. Yong, and M. Shoup, "Increased preoperative platelet count is associated with decreased survival after resection for adenocarcinoma of the pancreas," The American Journal of Surgery, vol. 189, no. 3, pp. 278-282, 2005.

[85] M. Ishizuka, H. Nagata, K. Takagi, Y. Iwasaki, and K. Kubota, "Combination of platelet count and neutrophil to lymphocyte ratio is a useful predictor of postoperative survival in patients with colorectal cancer," British Journal of Cancer, vol. 109, no. 2, pp. 401-407, 2013.

[86] S. Balta, S. Demirkol, M. Unlu, Z. Arslan, and T. Celik, "Comment on "a derived neutrophil to lymphocyte ratio predicts clinical outcome in stage II and III colon cancer patients"', British Journal of Cancer, vol. 109, no. 12, pp. 3125-3126, 2013.

[87] J. Szkandera, M. Pichler, M. Stotz, and A. Gerger, "Reply: comment on 'a derived neutrophil to lymphocyte ratio predicts clinical outcome in stage II and III colon cancer patients"', British Journal of Cancer, vol. 109, no. 12, pp. 3126-3127, 2013.

[88] P. Forget and M. de Kock, "Perspectives in anaesthesia for cancer surgery," Journal of Cancer Research and Clinical Oncology, vol. 140, no. 3, pp. 353-359, 2014.

[89] R. Motaghedi, J. J. Bae, S. G. Memtsoudis et al., "Association of obesity with inflammation and pain after total hip arthroplasty," Clinical Orthopaedics and Related Research, vol. 472, no. 5, pp. 1442-1448, 2014.

[90] D. Lacombe, S. Burock, and F. Meunier, "Academia-industry partnerships: are we ready for new models of partnership?: the point of view of the EORTC, an academic clinical cancer research organisation," European Journal of Cancer, vol. 49, no. 1, pp. 1-7, 2013.

[91] P. Forget, B. Tombal, J.-L. Scholtès et al., "Do intraoperative analgesics influence oncological outcomes after radical prostatectomy for prostate cancer?" European Journal of Anaesthesiology, vol. 28, no. 12, pp. 830-835, 2011.

[92] J. P. Cata, V. Gottumukkala, and D. I. Sessler, "How regional analgesia might reduce postoperative cancer recurrence," European Journal of Pain Supplements, vol. 5, no. 2, pp. 345-355, 2011.

[93] I. Bhatti, O. Peacock, G. Lloyd, M. Larvin, and R. I. Hall, "Preoperative hematologic markers as independent predictors of prognosis in resected pancreatic ductal adenocarcinoma: neutrophil-lymphocyte versus platelet-lymphocyte ratio," The American Journal of Surgery, vol. 200, no. 2, pp. 197-203, 2010.

[94] R. A. Smith, L. Bosonnet, M. Raraty et al., "Preoperative platelet-lymphocyte ratio is an independent significant prognostic marker in resected pancreatic ductal adenocarcinoma," The American Journal of Surgery, vol. 197, no. 4, pp. 466-472, 2009.

[95] N. Sakka, R. A. Smith, P. Whelan et al., "A preoperative prognostic score for resected pancreatic and periampullary neuroendocrine tumours," Pancreatology, vol. 9, no. 5, pp. 670676, 2009.

[96] R. A. Smith, P. Ghaneh, R. Sutton, M. Raraty, F. Campbell, and J. P. Neoptolemos, "Prognosis of resected ampullary adenocarcinoma by preoperative serum CA19-9 levels and plateletlymphocyte ratio," Journal of Gastrointestinal Surgery, vol. 12, no. 8, pp. 1422-1428, 2008.

[97] R. A. Smith, L. Bosonnet, P. Ghaneh et al., "The plateletlymphocyte ratio improves the predictive value of serum CA199 levels in determining patient selection for staging laparoscopy in suspected periampullary cancer," Surgery, vol. 143, no. 5, pp. 658-666, 2008. 


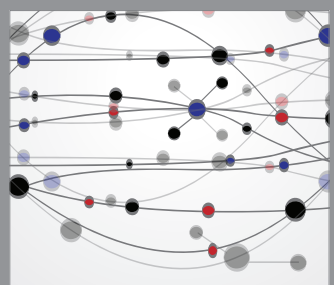

The Scientific World Journal
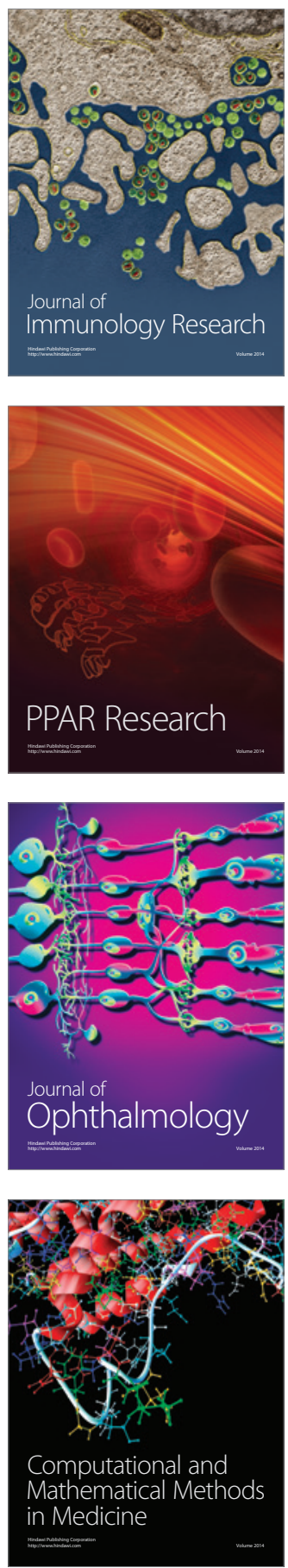

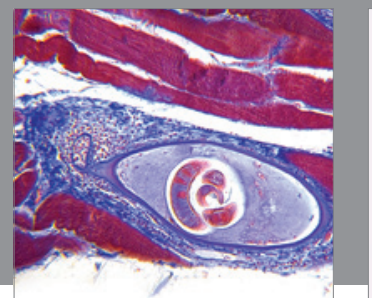

Gastroenterology

Research and Practice
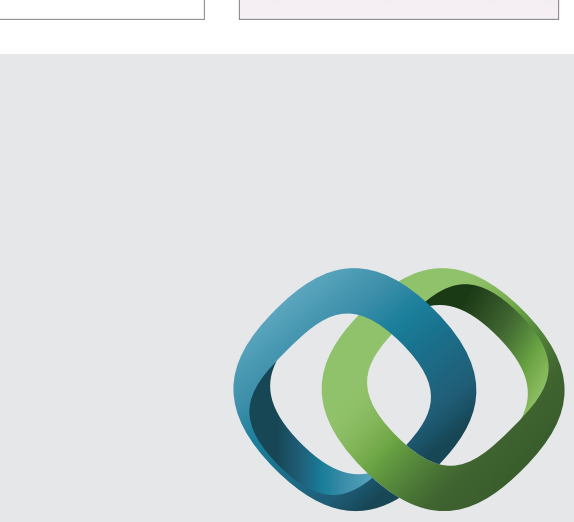

\section{Hindawi}

Submit your manuscripts at

http://www.hindawi.com
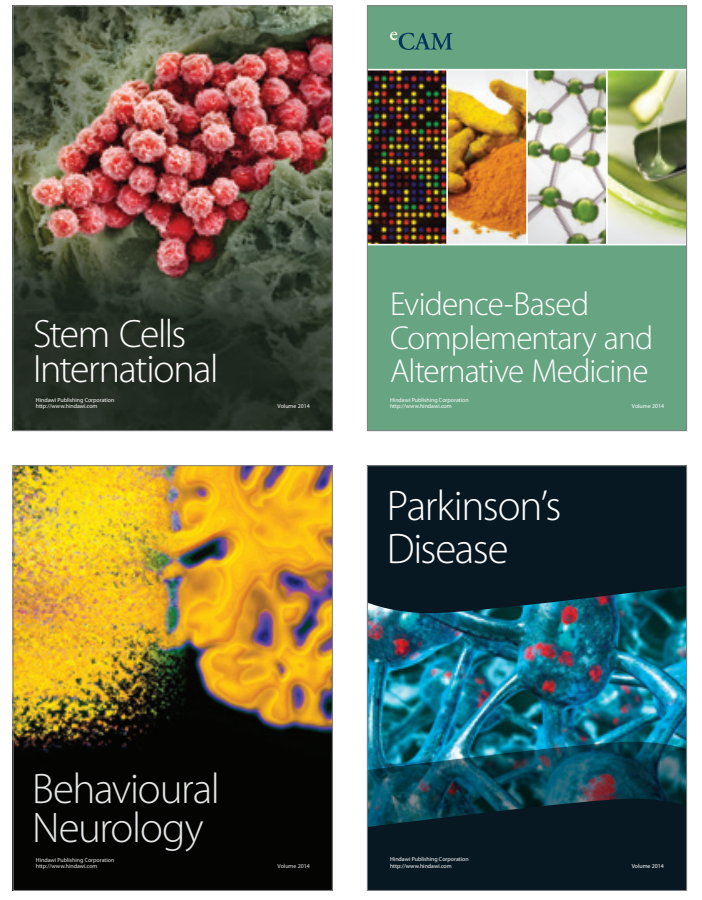
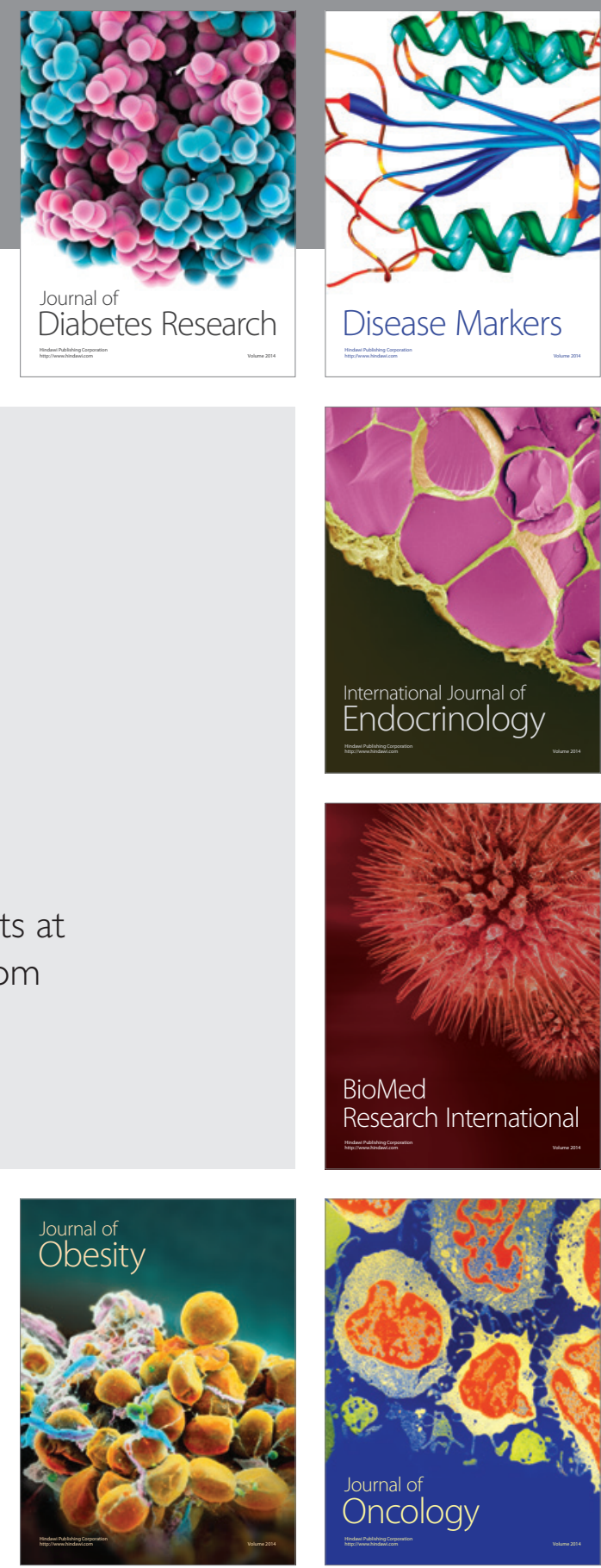

Disease Markers
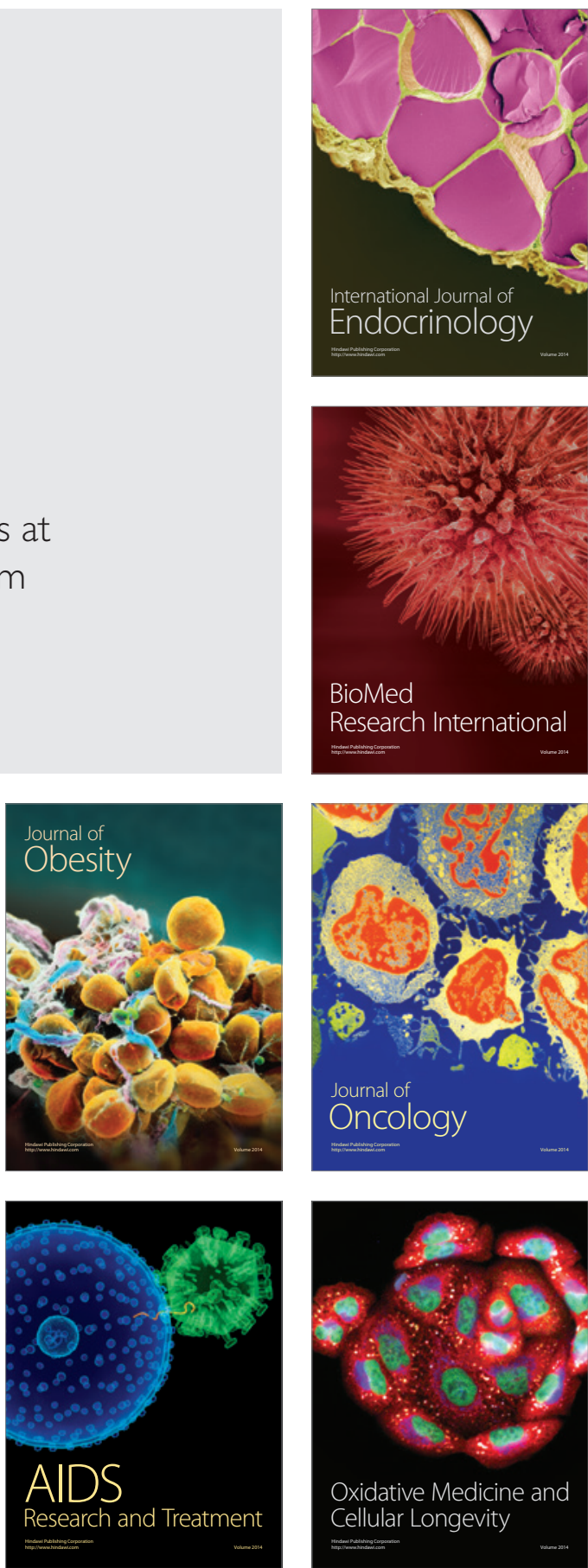